PSS $\quad \begin{aligned} & \text { PROCEEDINGS } \\ & \text { OF SCIENCE }\end{aligned}$

\title{
Ultra-fast Silicon Detectors
}

\author{
Abraham Seiden ${ }^{1}$ \\ University of California Santa Cruz \\ 1156 High Street, Santa Cruz, CA, United States \\ E-mail: aseiden@ucsc.edu
}

Ultra-fast silicon detectors are a recent detector type using high resistivity n-on-p wafers and including an extra implantation step to create a high field region that amplifies drifting electrons. The target gain is approximately a factor of ten, allowing high rate operation and good time resolution at the same time as good position resolution is achieved. The best time resolution is expected to be achieved for thin detectors, for example 50 microns thick sensors. This talk will cover how the ultra-fast detectors work, some measurements made to date, and performance expectation based on simulations. Also the extensive prototyping program recently launched to explore the choice of detector parameters will be described. The prototyping program should yield significant new information in the next six months and provide validation for the simulation program that has been developed.

24th International Workshop on Vertex Detectors -VERTEX2015-

1-5 June 2015

Santa Fe, New Mexico, USA

\section{${ }^{1}$ Speaker}




\section{Introduction}

Ultra-fast silicon detectors are a recent silicon detector development enabled by the advances in the use and fabrication of silicon sensors in the particle physics community. These sensors [1] are obtained using high resistivity $p$ type wafers with an $n$ implantation to form the junction and to collect electrons liberated by an ionizing particle passing through the sensor. One extra p-implantation step is required, providing a p-layer just under the n-layer, yielding a localized high-field region that amplifies the electrons drifting through the region. The goal for the amplification field is such that holes will not be amplified, avoiding a breakdown, and allowing dead-timeless operation. In practice this results in a target gain of $\sim 10$ and requires an amplification field of about $300 \mathrm{kV} / \mathrm{cm}$.

The goal for the Ultra-fast silicon detectors is to provide a sensor that measures particle arrival times with a resolution of $\sim 40$ psec, to complement the excellent position resolution possible using silicon. To achieve this goal, the electron drift-time within the sensor must be as short as possible, requiring reasonably large electric fields in the sensor bulk. The electron drift-velocity saturates at about $10^{7} \mathrm{~cm} / \mathrm{sec}$, requiring a drift field of about $20 \mathrm{kV} / \mathrm{cm}$. Figure 1 shows a pad detector fulfilling the various requirements for gain and electron drift speed for a bias voltage in the $500 \mathrm{~V}$ to $1000 \mathrm{~V}$ range.
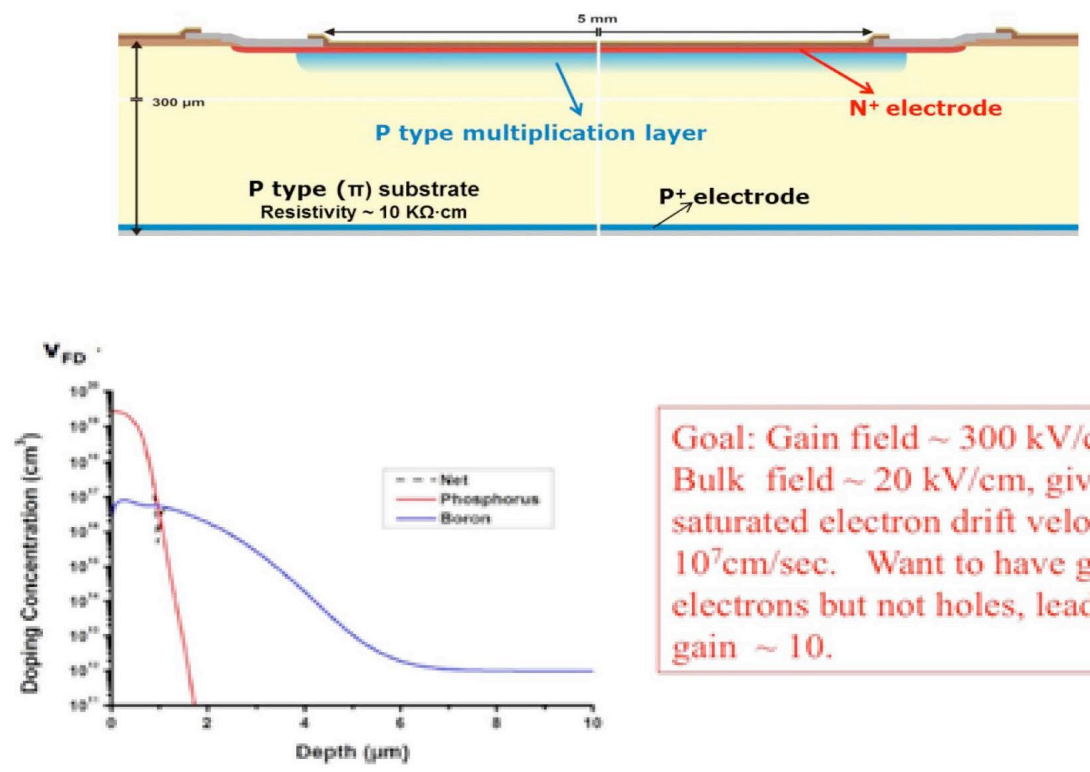

Goal: Gain field $\sim 300 \mathrm{kV} / \mathrm{cm}$.

Bulk field $\sim 20 \mathrm{kV} / \mathrm{cm}$, gives a saturated electron drift velocity $10^{7} \mathrm{~cm} / \mathrm{sec}$. Want to have gain for electrons but not holes, leads to gain $\sim 10$.

Fig. 1: Top: Cross section through a Ultra-fast sensor. Bottom: doping density within the device near the collection electrode. 
One thing to notice is that the gain-producing field, which comes mainly from the extra p-implantation, rides on the much smaller field created by the bias voltage on the detector. This makes the gain of the device weakly dependent on the detector voltage but requires good control of the doping density of the $\mathrm{p}$ implantation. This is illustrated in Figure 2 where the response to a fixed laser pulse is used to measure the gain versus the voltage for several sensors.

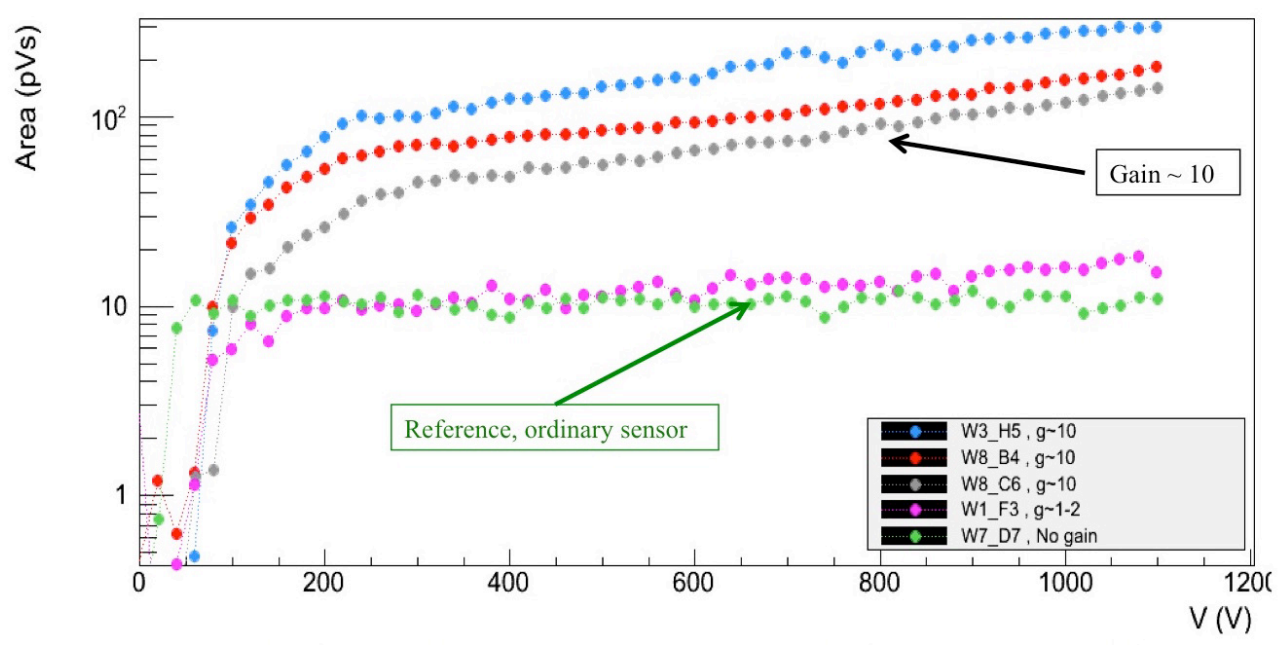

Fig. 2: Gain versus bias voltage for several $8 \mathrm{~mm}$ x $8 \mathrm{~mm}$ pad detectors of thickness equal to $285 \mathrm{~mm}$. Three sensors can be biased to have a gain of about 10, one has a very low gain and the last one for comparison is without gain.

Several other sensor parameters such as the sensor capacitance and detector thickness strongly affect the performance. The thickness determines the total charge collection time. To date only Ultra-fast pad detectors of thickness $\sim 300 \mathrm{~mm}$ have been fabricated and tested in a beam. We depend on a simulation program, Weightfield2 [2], to predict the performance of thinner detectors. This program indicates that a $50 \mathrm{~mm}$ thick sensor would provide much better timing resolution than a $300 \mathrm{~mm}$ sensor, getting us down to our target goal for timing accuracy. We will be testing this in the coming year as a program has been launched to produce and test thinner sensors [3].

\subsection{Basics of Time Measurement and Signal Characteristics}

The timing accuracy for a silicon sensor is determined by the pulse shape of the signal at the output of an amplifier. A traditional method for measuring the time is via a discriminator. For a discriminator with a fixed comparator threshold the timing accuracy is limited by time-walk (since signals with different pulse heights cross the comparator threshold at different times), electronics noise (jitter), and also the binning accuracy for 
the device that stores the time. The time-walk can be reduced by using a constant fraction discriminator, provided the signal peaks at a time approximately independent of its amplitude. An even more elaborate scheme would involve sampling the signal in small time bins to fit the start time. This can be used to eliminate time-walk and reduce jitter by averaging, but is very costly given the complexity, which makes it disfavored for a system with many channels.

To evaluate and compare sensor choices we use as a figure of merit the slew-rate of the signal (change in amplitude per unit time) after the amplifier. This depends on the sensor signal characteristics and the amplifier rise time $\left(\mathrm{t}_{\mathrm{r}}\right)$. To provide a good measurement we need both large and fast signals. These are enhanced by having gain in the detector.

The other important factor is the noise, for which the Equivalent Noise Charge (ENC) has terms which depend on the amplifier details and sensor characteristics. Looking at the terms which depend on the sensor characteristics, $\mathrm{ENC}^{2}$ has current noise terms for which $\mathrm{ENC}^{2} \sim$ (detector leakage current) times $\mathrm{t}_{\mathrm{r}}$ and voltage noise terms for which $\mathrm{ENC}^{2} \sim\left(\mathrm{C}_{\mathrm{det}}\right)^{2} / \mathrm{t}_{\mathrm{r}} \sim \mathrm{C}_{\mathrm{det}}$ for the case where $\mathrm{t}_{\mathrm{r}}$ is proportional to the detector capacitance, $\mathrm{C}_{\text {det }}$. To minimize the voltage noise we need to keep the detector capacitance small, which also minimizes the rise time, reducing the current noise and maintaining a large slew-rate. All of these effects are included in the simulation program, referenced earlier $[2]$.

To understand better the role of the detector thickness we look at the dependence of signal timing characteristics on the thickness and gain for a pad detector with a saturated electron drift velocity. For a conventional detector we compare the signal for both a thin and thick detector. The rise time and slew-rate are approximately identical initially. The thicker detector has a larger integrated signal but this is due to a longer signal. This comes about because the charge deposited in the thicker sensor leads to more moving charges, however the weight-field is proportional to the inverse of the thickness, so the two cancel out at the beginning of the signal. This is shown schematically in Figure 3. 


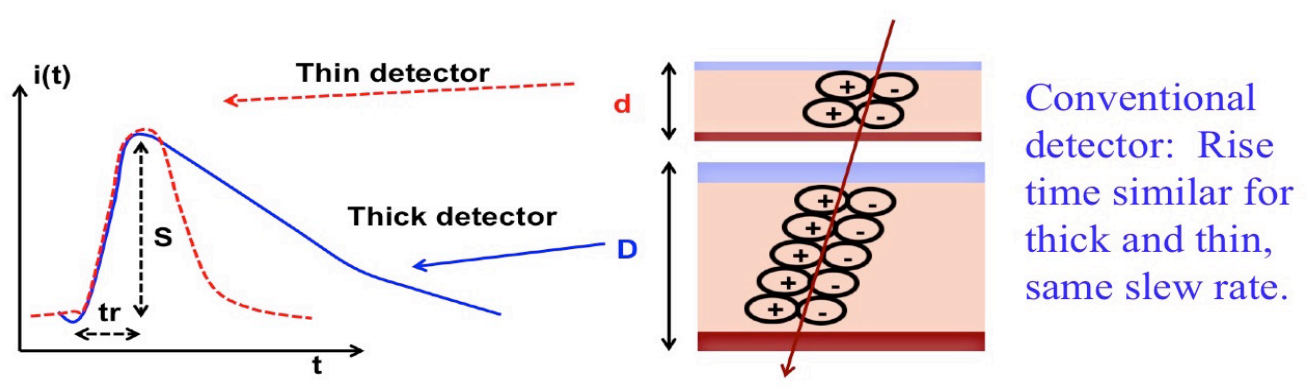

Fig. 3: Expected Signal for a Thin and Thick Detector without gain.

For a sensor with gain the rise time and slew-rate depend strongly on the detector thickness. This situation is shown schematically in Figure 4. In particular the maximum signal occurs when all the electrons have passed through the gain layer providing the large number of drifting holes resulting from the gain. The holes are slower than the electrons so they are all drifting still when the last electron is collected. This results in a pulse whose peak value is proportional to the gain and which occurs at a time corresponding to the full electron collection time, which is proportional to the detector thickness. This provides a stable peak-time, which depends only on the detector thickness and electron drift velocity as long as the weighting field is constant. Landau fluctuations affect the detailed shape of the pulse and the maximum pulse height but not the location in time of the peak. Important goals for the electronics are to not significantly couple Landau fluctuations through shaping into a peak-time location for the shaped signal and also to avoid reducing the slew-rate. These require a sufficiently fast amplifier.

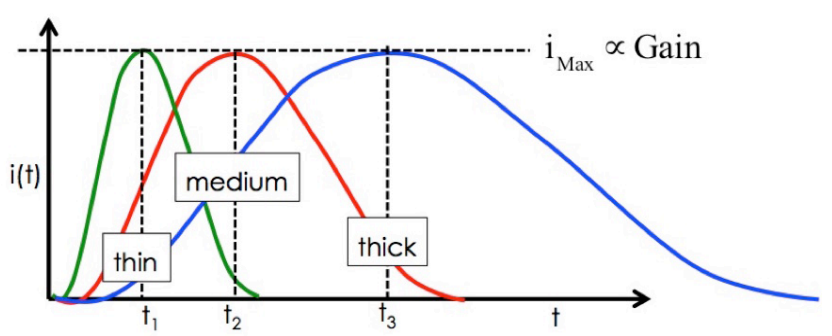
Detector with a gain
of 10: Rise time (and
slew-rate) are
different. Rise time
electron collection
time, is proportional
to detector thickness.

Fig. 4: Signal characteristics for three thicknesses of sensor, each with the same gain.

Using the Weightfield2 program, we show in Figure 5 the expected change in slew-rate versus detector thickness and gain for a minimum ionizing particle passing through sensors of varying thickness. The $50 \mathrm{~mm}$ sensor with a gain of 10 has an increase in slew-rate by a factor of 3.5 compared to a $300 \mathrm{~mm}$ thick sensor with no gain. This should translate into an improved ability to do timing. 


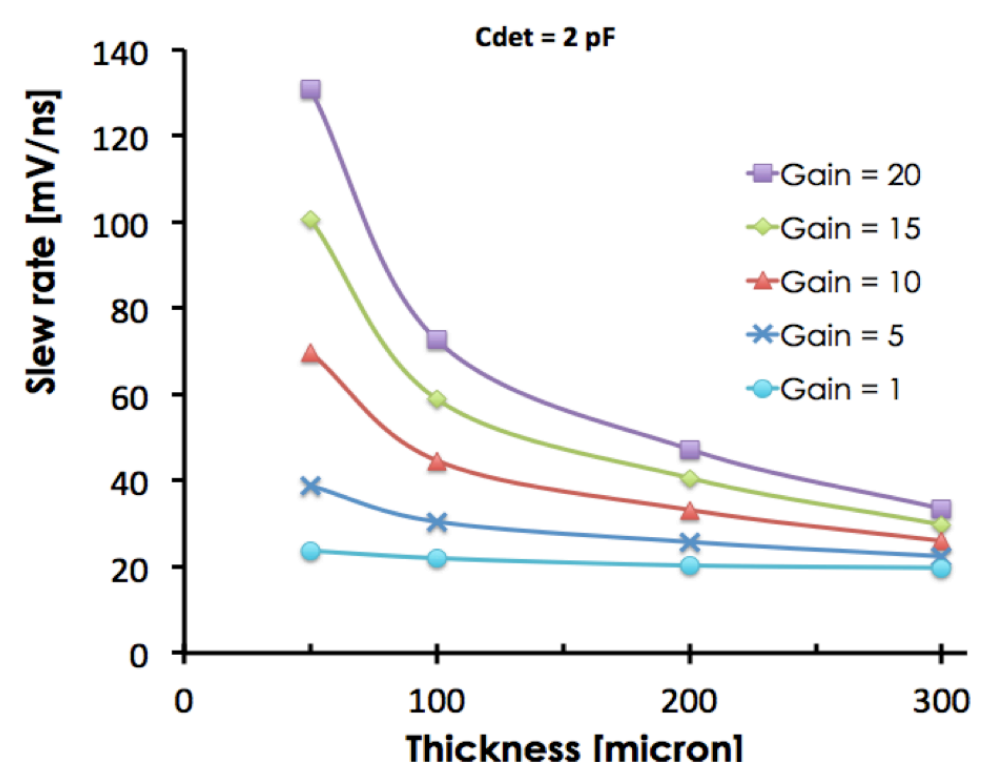

Fig. 5: Expected slew-rate for sensors of varying thickness and gain.

The simulation can be used to look at the components making up the signal for the 50 $\mathrm{mm}$ thick sensor, as shown in Figure 6. The primary electrons are collected in $0.5 \mathrm{nsec}$ and the signal peaks in $0.5 \mathrm{nsec}$, yielding a very large slew-rate.

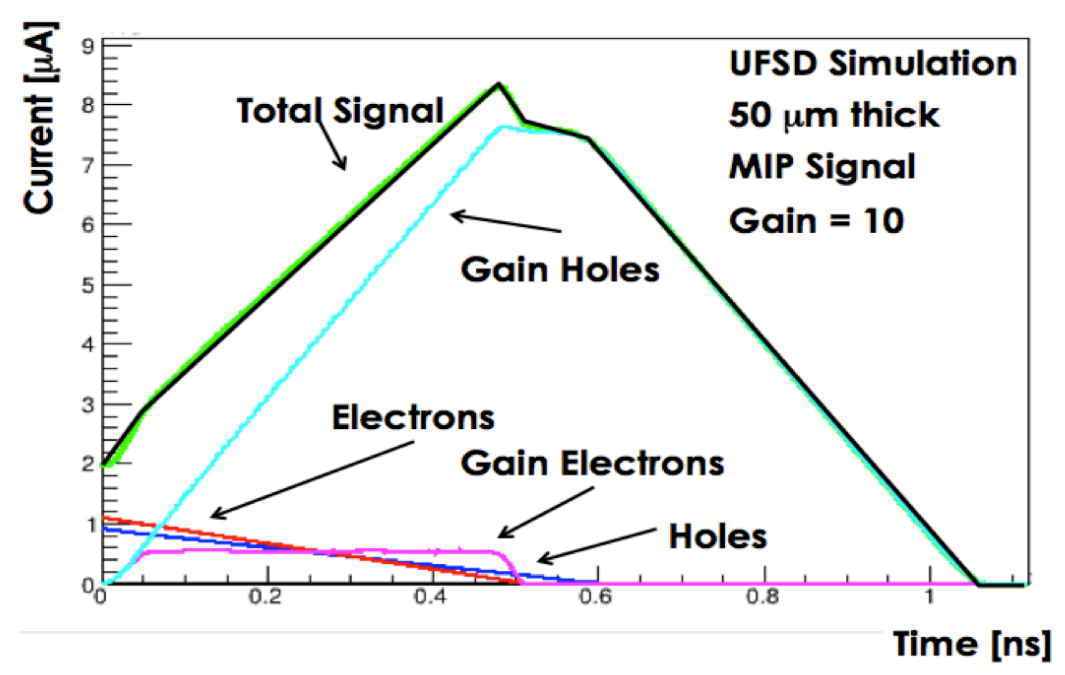

Fig. 6: Signal components and total signal for a $50 \mathrm{~mm}$ thick sensor with gain of 10 . 


\subsubsection{Tests of $300 \mathrm{~mm}$ thick pad detectors in Beam and using Lasers}

Data for pad detectors with gain were collected at two beam tests. In addition, a laser system has also been used to generate a very controlled signal in the detectors offering a chance to separately evaluate some of the detector characteristics. The first beam test was at Frascati using $500 \mathrm{MeV} / \mathrm{c}$ electrons while the second beam test was at CERN using a $120 \mathrm{GeV} / \mathrm{c}$ pion beam. The detector used at CERN had a capacitance of about 11 $\mathrm{pF}$, a gain of about 10, and run at $1000 \mathrm{~V}$. Scanning the detector with a laser indicated that the gain is constant over the pad face to about 3\%. We look below at results from the CERN beam test. Both beam tests gave very consistent results for timing resolution.

For the CERN beam test the start time was provided by a fast scintillator with SiPM readout with an estimated time jitter of $50 \mathrm{psec}$. The detector signals were amplified with a broad band amplifier with $50 \mathrm{ohm}$ input impedance (made by Civitek) and read into a $2.5 \mathrm{GHz}$ digital scope. For every trigger, the digitized pulse was recorded for 100 nsec in 50 psec steps and stored in a computer. The data were then analyzed off-line. The data had a small amount of high frequency pickup. To minimize the effect of this, we smoothed the signal by using a running 21 bin average. With the signal binning of $50 \mathrm{psec}$, the averaging amounts to a $-1 / 2$ to $+1 / 2$ nsec average. Figure 7 shows the distribution of pulse heights for the triggers that had a particle passing through the gain region of the pad detector. Visible are the single particle (1 MIP) signals and also a small number of events with multiple particles, with two particles (2 MIPs) most prominent. It's important to note that the data acquisition system (amplifier and oscilloscope) does not significantly affect the initial current pulse shape, providing an accurate picture of the signal dynamics.

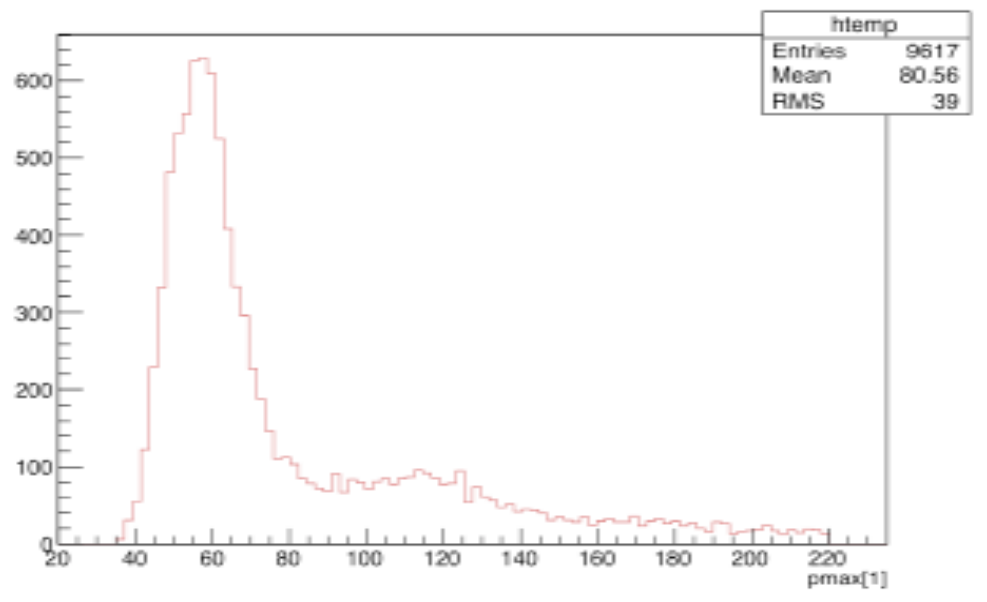

Fig. 7: Pulse height distribution in $\mathrm{mV}$ for events in the CERN beam test. 
We have examined several algorithms for measuring the time of the particle passage: straight line fits to two fixed pulse heights extrapolated to give the start time, and also constant fraction measurements, with time evaluated at various values for the fraction of the peak height. For our specific experimental set-up, the constant fraction techniques works best with the fraction $\sim 15 \%$ to $20 \%$ giving comparable results. Figure 8 shows the RMS and mean value for the time measurement for a $20 \%$ constant fraction in small bins in pulse height. From the Figure, we see that this technique has eliminated the time walk of a fixed pulse height measurement and the resolution improves as the pulse height increases as expected from an increased slew-rate.

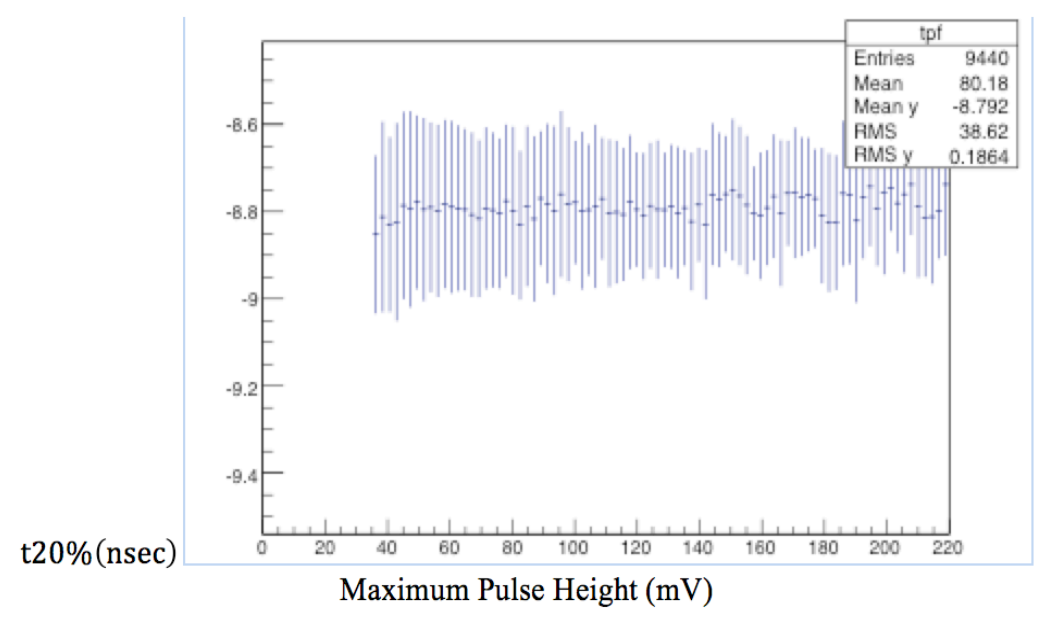

Fig. 8: RMS timing variation and mean value of time for a $20 \%$ constant fraction time measurement in pulse height bins.

This result, as well as the earlier discussion regarding the expectation of a very stable location in time of the sensor pulse peak, suggests that dividing each pulse by its maximum value will give a scaled pulse shape that can be used to isolate any unusual pulses and to see directly what different choices of constant fraction will give. The scaled pulses each have a maximum value of 1 but otherwise are distinct, with the pulse height scale now just the fraction of the maximum. This calculation results in pulse shapes shown in Figure 9a and 9b. For Figure 9a pulses that have a maximum between $55 \mathrm{mV}$ and $65 \mathrm{mV}$ (1 MIP region) have been chosen at random and overlaid so it can be seen how much they vary. Figure $9 \mathrm{~b}$ is the analogous plot where pulses with maxima between $110 \mathrm{mV}$ and $130 \mathrm{mV}$ (2 MIP region) have been randomly selected. The pulses are very similar, so it is clear why a constant fraction algorithm will work rather well. The baseline fluctuations for the 2 MIP case are half as large as for 1 MIP since the maximum pulse height is typically twice as large as for 1 MIP and all pulse bins are divided by the maximum. It can be seen that the pulse timing fluctuations around half the height are subsequently reduced when the pulses come together at the maximum. 

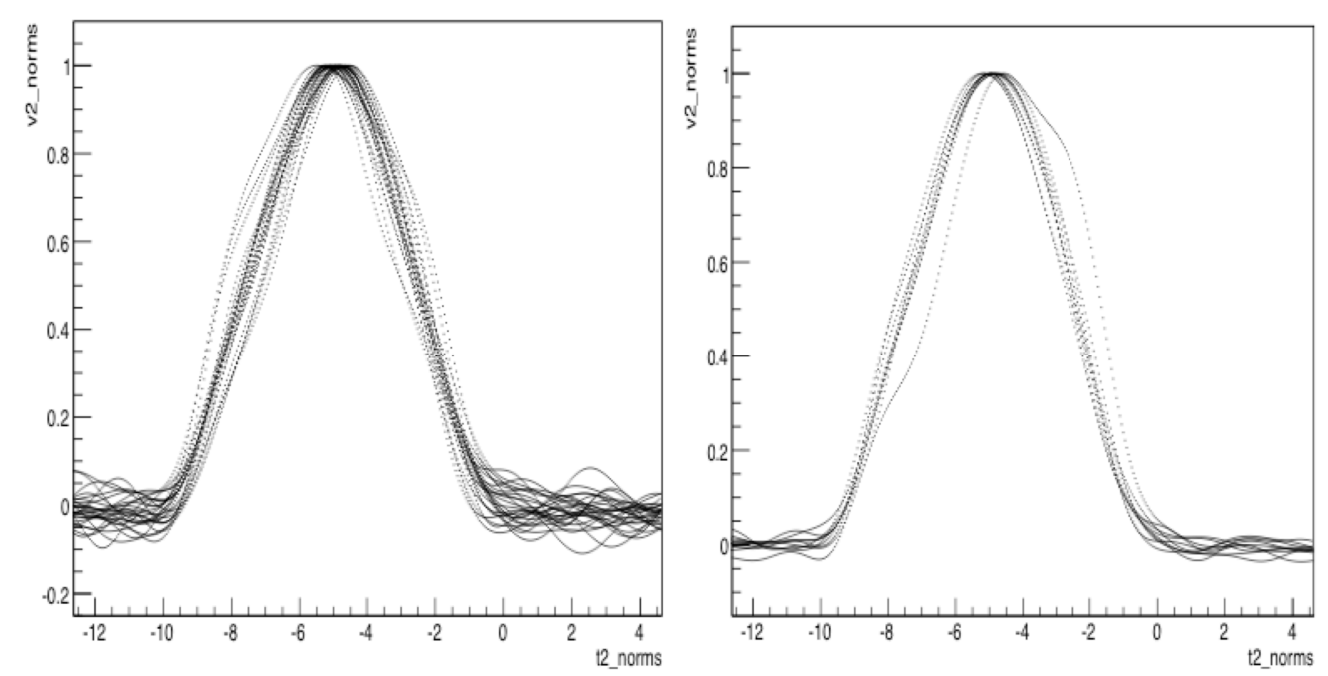

Fig. 9a and b: Overlay of rescaled pulses for those near 1 MIP (9a) and near 2 MIP (9b).

The rising pulses in Figure 9 are approximately linear with time prior to reaching a maximum. This suggests describing them by an equation:

$$
\mathrm{t}=\mathrm{t}_{0}+\mathrm{m}_{0}\left(\mathrm{x} / \mathrm{x}_{\mathrm{Max}}\right)
$$

where $\mathrm{x}$ is the pulse height, $\mathrm{x}_{\mathrm{Max}}$ the maximum pulse height, and $\mathrm{t}_{0}$ and $\mathrm{m}_{0}$ are constants. For the family of pulses $t_{0}$ is approximately $-9.6 \mathrm{nsec}$ (a number determined by the trigger setup as well as the pulse start time) and $\mathrm{m}_{0}$ (now characteristic of the signal) is approximately 4 nsec. This equation allows a direct calculation of the expected limitation in the time resolution due to jitter. Assuming independent and equal variations in $\mathrm{x}$ and $\mathrm{x}_{\text {Max }}$ due to jitter (given by $\mathrm{s}_{\mathrm{x}}$ ) and a constant fraction $=\mathrm{f}$, this equation yields a timing resolution due to jitter given by:

$$
\mathrm{o}_{\mathrm{t}}=\mathrm{o}_{\mathrm{x}}\left(\mathrm{m}_{0} / \mathrm{x}_{\mathrm{Max}}\right) \operatorname{sqrt}\left(1+\mathrm{f}^{2}\right)
$$

The expression is not too sensitive to the choice of constant fraction. $\mathrm{x}_{\mathrm{Max}} / \mathrm{m}_{0}$ is the slew- rate, advocated earlier as a good figure of merit for comparing detector choices. 
The jitter term $s_{x}$ depends on the choice of electronics and detector. We can evaluate $s_{x}$ from the data directly by looking at the region in time prior to the pulse start. The pulse height distribution at a given time prior to the signal start is well described by a Gaussian with a value: $s_{x}=1.9 \mathrm{mV}$. For a $60 \mathrm{mV}$ pulse $(1 \mathrm{MIP})$ this gives a $\mathrm{s}_{\mathrm{t}}=130$ psec for small $\mathrm{f}$ values.

In addition to jitter, the pulses have variations in the signal shape. In this case the effect of the choice of fraction $\mathrm{f}$ has a larger impact. A small value for $\mathrm{f}(<20 \%)$ results in a better resolution by about $20 \%$ than just using the time of the maximum. A value of $f$ of $50 \%$ to $80 \%$ results in worse resolution by $30 \%$ than choosing the time of the maximum.

The constant fraction method, taking a fraction $=15 \%$, gives a resolution for 1 MIP of $189 \mathrm{psec}$, and $129 \mathrm{psec}$ for 2 MIPs. We can estimate several of the contributors to the resolution. We estimate that the start time variation and finite binning give a 50 psec contribution to the error. The electronics jitter, calculated above, is about $130 \mathrm{psec}$ for 1 MIP and 65 psec for 2 MIPs. Assuming errors in quadrature we can remove these two sources of error, leaving 139 psec for 1 MIP and 100 psec for 2 MIPs from other sources, including pulse shape fluctuations. The value for the time resolution from electronics jitter can be separately measured by looking at detector signals generated by a laser whose charge deposition is very uniform and equal to 1 MIP. This measurement gives excellent agreement with the value extracted for the jitter in the beam-test. The measured points, along with predictions made for detectors with comparable capacitance to our beam-test detector are shown in Figure 10. Also shown are predictions for thinner detectors and smaller pad sizes.

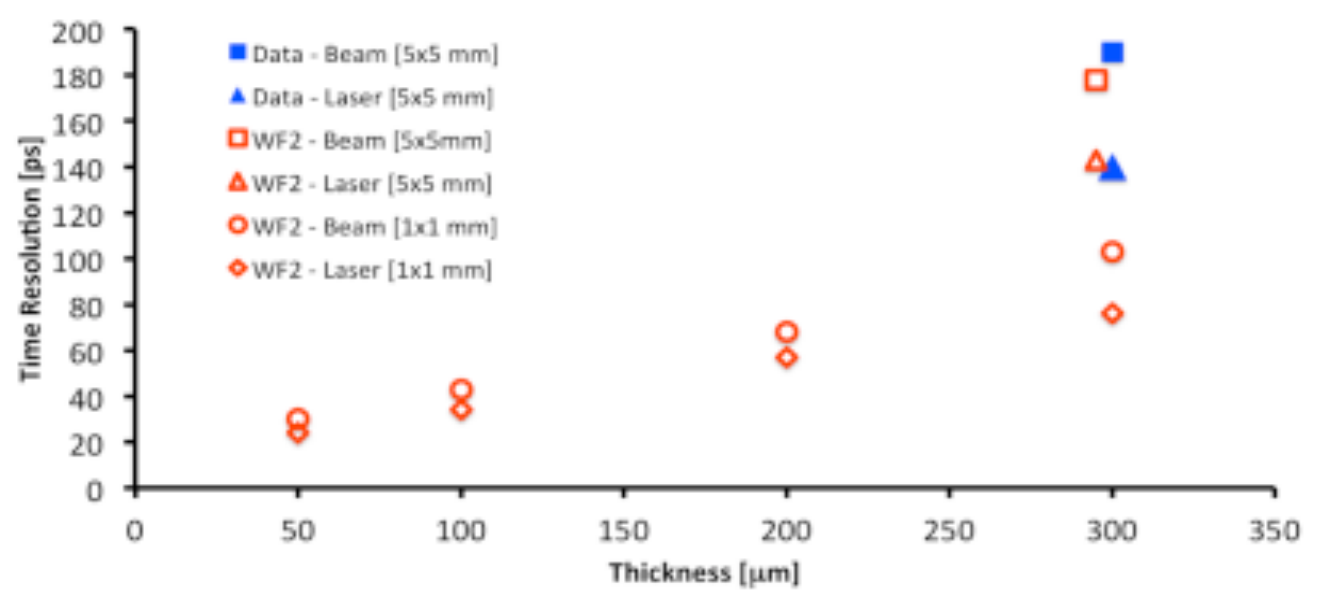

Fig. 10: Measurements (blue) and some predictions (red) for time resolution. 


\subsubsection{Measurements for Additional Pad Sensors}

A new set of sensors have been delivered [3], allowing further study of the use of sensors with gain to precisely measure times. These sensors are still $300 \mathrm{~mm}$ thick but now with smaller pad-size, $3 \mathrm{~mm}$ by $3 \mathrm{~mm}$, resulting in a significant reduction in detector capacitance, which is now $\sim 2 \mathrm{pF}$. Some predictions for sensors with smaller capacitance are shown in Figure 10, to be compared to the results above. Results from a beam test of these devices should be available soon. We look at some measurements [4] made using a laser, so the results quantify the effect of jitter. Measurements have been made with both the broad band (BB) amplifier and a charge sensitive amplifier (CSA, also by Civitek) with 4 nsec integration time. Figure 11 shows the gain versus voltage as well as the measured resolution of the detector for a laser-simulated 1 MIP signal for the CSA and a few points for the BB. The CSA, which has lower ENC than the BB, gives a lower jitter but at the cost of a longer shaping time. The latter has no penalty since the laser signal is constant from pulse-to-pulse. The resolution, for a gain of 6 , is about $60 \mathrm{psec}$ for the CSA. For the BB it is about $100 \mathrm{psec}$, showing some improvement due to lower capacitance for this detector, compared to the measurement for a larger pad in Figure 10.

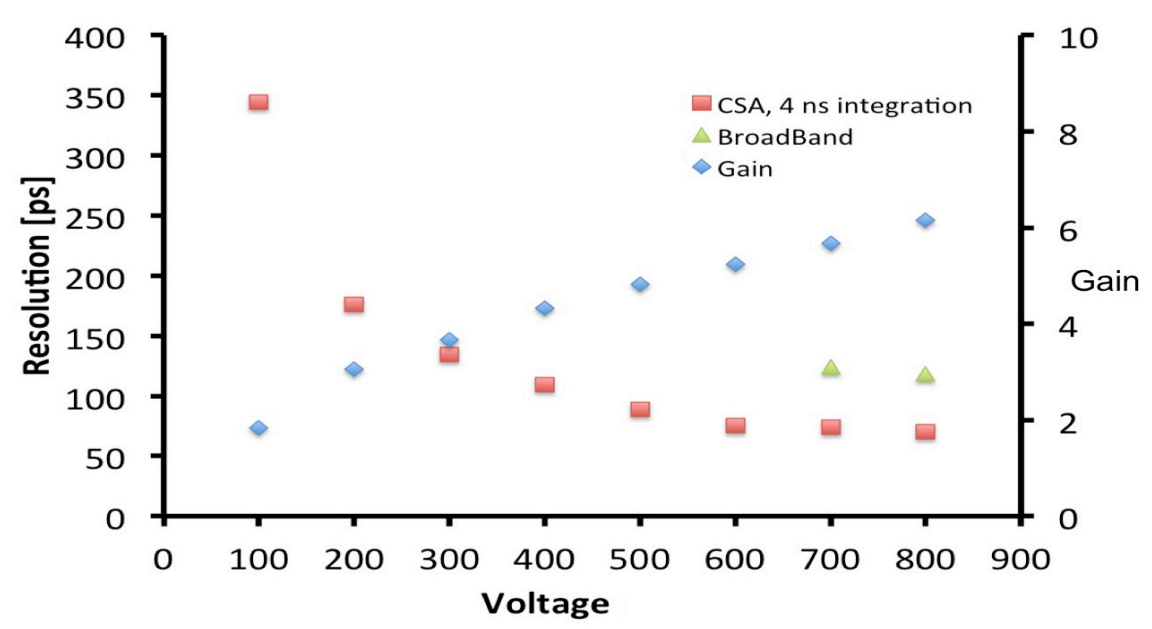

Fig. 11: Resolution and gain versus voltage for a $3 \mathrm{~mm} \times 3 \mathrm{~mm}$ pad detector, using laser generated MIP equivalent signals.

Using the laser we can also study the effect of larger pulse heights by increasing the laser power. Figure 12 shows the measured resolution with the CSA for laser signals of various amplitudes expressed in MIP equivalents. The gain is 6 for the detector with gain. Also shown is the measured resolution for a conventional diode run at large voltage ( 800 volts) for comparison. The measured resolution tends to level-off at large pulse heights, which probably reflects the jitter in the start of the laser signal. The plot 
indicates the potential for very accurate timing measurement in a shower of particles provided they all hit the detector at the same time.

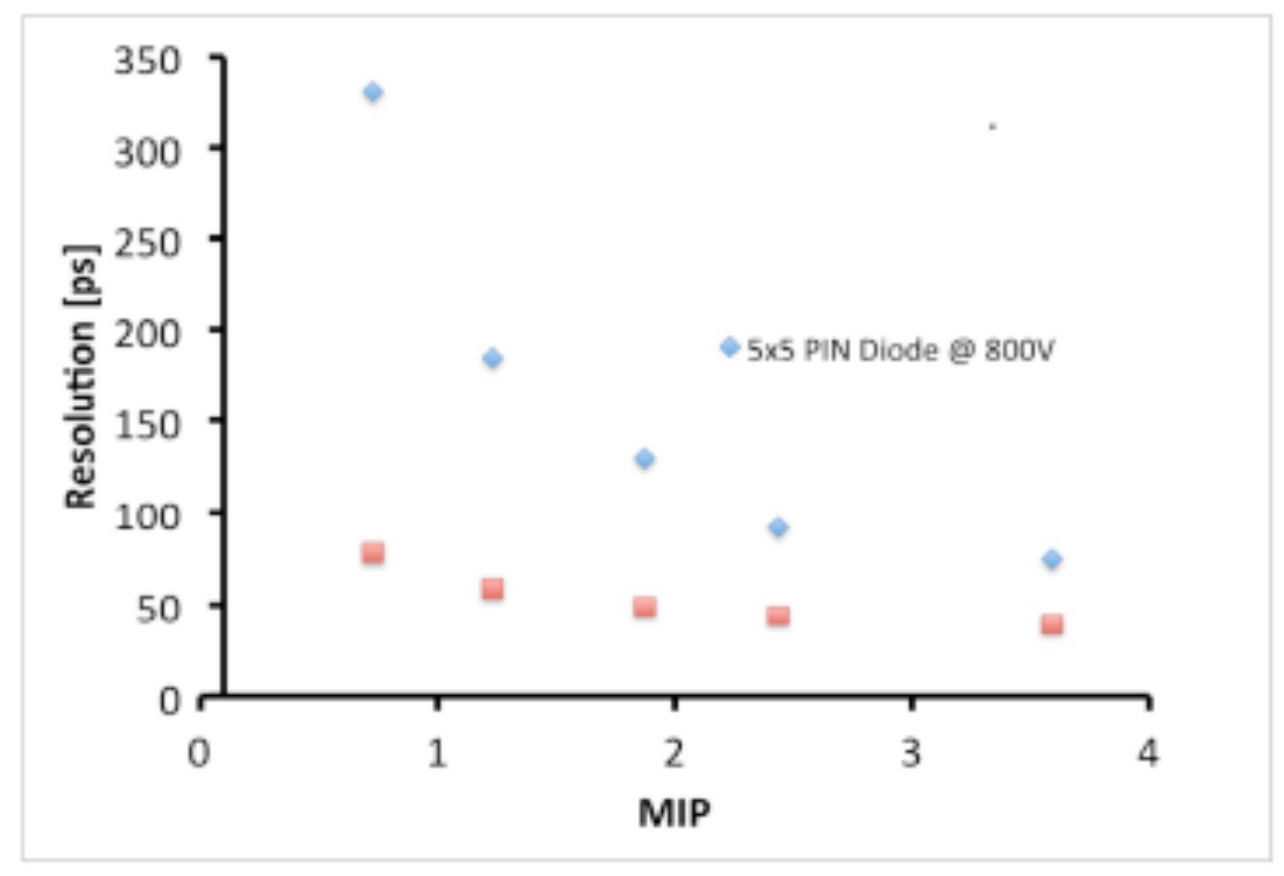

Fig.12: Resolution for $3 \mathrm{~mm} \times 3 \mathrm{~mm}$ pad detector with gain of 6 for signals of various amplitudes generated by a laser (red). For comparison, results for a conventional diode are also shown (blue).

\section{Applications for Pad Detector}

The $\sim 3 \mathrm{~mm} \times 3 \mathrm{~mm}$ pad detectors should allow several types of interesting application. The first is in medical physics using good time of flight measurements. An example is pCT (proton computed tomography) for cancer treatment using proton beams. The use of protons has the advantage that the dose is delivered near the end of the range of the beam, allowing precise targeting of a tumor, but requiring therefore good localization. Using protons the tumor area can be mapped prior to treatment. This requires detection of the scattered protons using some type of detector telescope which measures both trajectories and proton energy. One approach has been to measure the trajectory with silicon detectors and the energy in a subsequent range detector. The use of time-offlight measured by Ultra-fast detectors would allow a big improvement in the energy measurement, with cleaner signals and higher rates possible. These would both be advantageous for patients being scanned for eventual treatment.

An application in the particle physics area would be to build a forward timing detector at the LHC. This detector could be made of several planes at very forward rapidities, 
located a few meters from the interaction region. One could build each plane using 6 inch wafers fabricated to have gain. Each wafer would contain many unit cell patterns, with the unit cell shown in Figure 13. The unit cell is made of four groups of $3 \mathrm{~mm} \times 3 \mathrm{~mm}$ pads read out by one four-channel chip positioned at the intersection point of the pads. The connections are made by wire bonding and a flex circuit (not shown) would need to carry both voltages and signals. To keep the dead-area between pads very small, discrete bias resistors would also be wire-bonded to each pad. To reach the desired resolution of $40 \mathrm{psec}$ the detectors would be 50 micron thick. This year we plan to be testing such detectors [3].
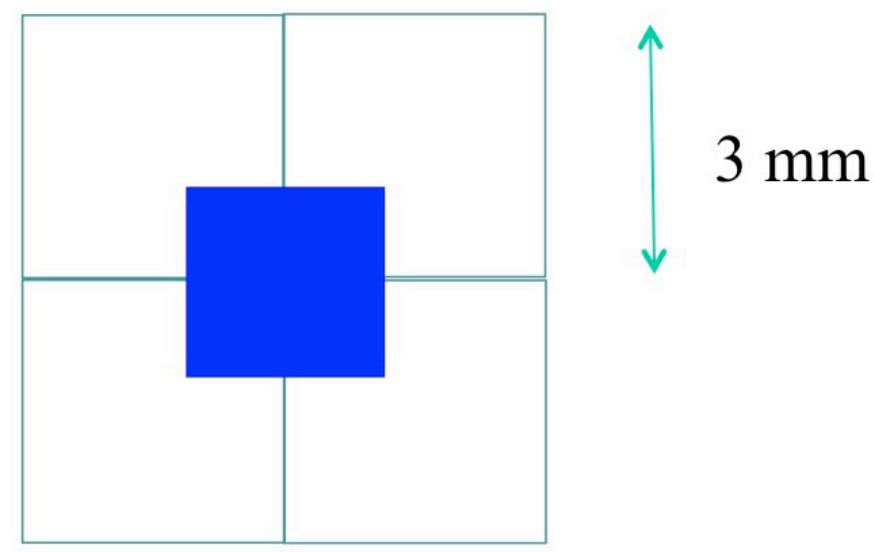

Fig. 13: Unit cell made of four Ultra-fast detectors read out by one 4 channel chip (in blue).

\section{Developing a Fine Grained Pixel Detector}

To increase the number of potential applications it would be advantageous to build the gain mechanism into a sensor with fine-grained pixels that can be individually readout. A major challenge is maintaining the very constant field generated by the gain layer and keeping a constant weighting-field. The nicest solution would be to leave the detector as is and pixelate the metallization layer that the electronics connects to. This can work if the charge is held locally for sufficient time by the n-implant. It is however difficult to have a sufficiently high sheet-resistance for this to work. We are instead first looking at the situation where the side of the detector containing the gain layer is unchanged, keeping the very constant field and putting the charge collecting pixels on the other side of the detector. We plan to test such detectors this coming year [5].

\section{Conclusions}

We have presented the key concepts underlying the Ultra-fast silicon detector. First timing measurements for a $300 \mathrm{~mm}$ thick sensor agree with expectations and allow a 
check that the signal characteristics, such as a rather well defined peak time, can be seen in the data. This year we plan to explore in more depth the behavior of Ultra-fast sensors as a function of detector thickness and capacitance. Important characteristics to validate are the slew-rate versus thickness and resultant time resolution that can be achieved. For pad detectors a number of potential applications can already be identified. Studies of devices with more finely pixelated charge collection will also begin soon.

\section{Acknowledgements}

This work was done under the umbrella of the RD50 CERN based silicon detector collaboration. Special thanks are due to the groups at:

UC Santa Cruz, Torino, Barcelona and CNM, Trento and FBK, Ljubljana.

\section{References}

[11] H.-W. Sadrozinski, et al., Ultra-fast silicon detectors, Nucl. Instrum. Meth. A730 ए(2013) 226.

$>$ @H.-W. Sadrozinski, et al., Ultra-fast silicon detectors, Nucl. Instrum. Meth. A765

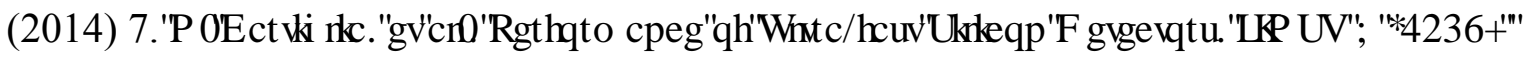

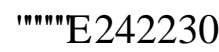

[0 @ (a) Cenna, et al., Weightfield2 a fast simulator for silicon and diamond solid state Idetectors, Nucl. Instrum. Meth. A796 (2015) 149

[0] G. Pellegrini, et al., Technology developments and first measurements of Low Gain \Avalanche Detectors (LGAD) for high energy physics applications, Nucl. Instrum. Meth. A765 (2014) 12.

These measurements were made by the group at the University of Torino, and were[SLRYIGHET 11 IFRORI\&DWN (DD)

[5] [G.-F. Dalla Betta, et al., Design and TCAD simulation of double-sided pixilated low gain avalanche detectors, Nucl. Instrum. Meth. A796 (2015) 154 